\title{
Optimal Scheduling for Emptying a Wireless Network: Solution Characterization, Applications, Including Deadline Constraints
}

Qing He, Di Yuan and Anthony Ephremides

The self-archived postprint version of this journal article is available at Linköping University Institutional Repository (DiVA):

http://urn.kb.se/resolve?urn=urn:nbn:se:liu:diva-165010

N.B.: When citing this work, cite the original publication.

He, Q., Yuan, Di, Ephremides, A., (2020), Optimal Scheduling for Emptying a Wireless Network: Solution Characterization, Applications, Including Deadline Constraints, IEEE Transactions on Information Theory, 66(3), 1882-1892. https://doi.org/10.1109/TIT.2019.2957800

Original publication available at:

https://doi.org/10.1109/TIT.2019.2957800

Copyright: Institute of Electrical and Electronics Engineers (IEEE)

http://www.ieee.org/index.html

(C)2020 IEEE. Personal use of this material is permitted. However, permission to reprint/republish this material for advertising or promotional purposes or for creating new collective works for resale or redistribution to servers or lists, or to reuse any copyrighted component of this work in other works must be obtained from the IEEE. 


\title{
Optimal Scheduling for Emptying a Wireless Network: Solution Characterization, Applications, Including Deadline Constraints
}

\author{
Qing He, Member, IEEE, Di Yuan, Senior Member, IEEE, and Anthony Ephremides, Life Fellow, IEEE
}

\begin{abstract}
Link scheduling, i.e., which links should transmit together and for how long, has been and remains a cornerstone optimization problem in wireless networking. In minimum-time scheduling, the task is to minimize the amount of time before emptying the data demand residing at the source nodes. We derive a complete structural characterization of the solution that unifies and significantly extends the known results. First, we approach link scheduling with a general system model without restrictions on the shape of the achievable rate region. Then, we give and prove a solution characterization of optimality that is conceptually simple yet powerful. We demonstrate several applications of this characterization for analysis of optimality and problem tractability. Next, we consider a significant extension by including deadline constraints, under which optimal scheduling becomes much more complex. Yet, we show how our formulation yields a solution description for that problem as well.
\end{abstract}

Index Terms-deadline, optimality, scheduling, wireless networks.

\section{INTRODUCTION}

A fundamental aspect of access coordination in wireless networks is link scheduling. Due to the broadcast nature of wireless media, a set of links that are concurrently active cause interference to each other. The transmission rate of each active link in the set depends on the composition of that set as well as other factors such as power, coding and modulation scheme, and so on. The scheduling problem amounts to deciding which links should simultaneously transmit and for how long so as to attain some optimality goal. In this paper, we consider the "empty-the-network" scheduling problem where the task is to empty given amounts of data residing at the transmitters. This type of scheduling problem has been extensively studied under various setups. Unlike most of the previous work, however, our objective is not to address a specific model or one algorithmic refinement. The objective is to gain better understanding of

Q. He is with the School of Electrical Engineering and Computer Science, KTH Royal Institute of Technology, Stockholm, SE-11428, Sweden. (e-mail: qhe@kth.se)

D. Yuan is with the Department of Science and Technology, Linköping University, Norrköping SE-60174, Sweden. (e-mail: di.yuan@liu.se)

A. Ephremides is with the Department of Electrical and Computer Engineering, University of Maryland, College Park, MD 20742 USA. (e-mail: etony@umd.edu)

Part of this paper was presented at the 2016 and the 2017 IEEE International Symposium on Information Theory.

Copyright (c) 2017 IEEE. Personal use of this material is permitted However, permission to use this material for any other purposes must be obtained from the IEEE by sending a request to pubs-permissions@ieee.org. the generic structure and optimality, unifying and expanding the knowledge on more specific problem setups, with generalization to new problem extensions. To this end, we study the scheduling problem in a general form, such that the formulation can accommodate any physical layer properties, interference model, channel behavior, or other implementation details, e.g., power control. We then derive and prove a series of results, to establish the fact that minimum-time scheduling is equivalent to maximum scaling of the link demand vector over the convex hull of the rate region. The characterization is conceptually simple yet powerful, to understand, within a unified framework, why optimal scheduling of specific setups exhibit their respective properties, and thereby to yield more generalized results. Then, we introduce a significant extension to our problem by including link-specific deadline constraints, under which little has been known for optimal scheduling. Leveraging our viewpoint of optimality characterization, we characterize optimal scheduling in the presence of deadline.

\section{RELATED WORK}

A rich amount of literature is available for the scheduling problem (see, for example, the surveys [1]-[3] and the references therein). Notable amongst earlier works is the spatial time division multiple access (STDMA) scheme, in which multiple non-conflicting links are assigned to each time slot [4]. The problem is of combinatorial nature and has been proved to be hard in general [5]. Under the, so called, protocol model, graph-based algorithms employing implicit or explicit coloring strategies were widely used (e.g., [6], [7]). Later on, the scheduling problem was studied under the interference model [8]. This approach incorporates the effect of interference on the achievable rates. Further developments consist in integrating the problems of routing, scheduling, and physical layer effects [1], [9]. In [10], the authors show that the scheduling problem can be represented using a set-covering formulation. The scheduling problem under the interference model is proved to be NP-hard with arbitrary gain and geometric-based gain, in [10] and [11], respectively. Many heuristic and approximation algorithms have been investigated. In [12], a column-generation based solution method is proposed. The minimum-time scheduling with fixed link rates is studied in [13], where each link subset, once selected, transmits with a given rate vector. In our current work, we focus on the generalization, where each link subset may have multiple, or even infinitely many, rate vectors that 
are subject to selection, to account for systems deploying various types of mechanisms to enable new dimensions of freedom in link rates. For the general formulation, the analysis in [13] using properties of linear programming does not apply. Moreover, [13] does not address any deadline constraints.

The "empty-the-network" scheduling problem differs from the stability problem, which targets to maintain the stability of the queues in the network and maximize some utility function [2]. The classic back-pressure algorithm [14] or driftplus-penalty algorithm [3] applies to the stability problem. The relation between system stability and minimum evacuation time in a time-slotted system with input queues of infinite length is investigated in [15]. In this paper we do not consider arrivals or queueing for stability. Hence the scheduling problem that we consider is different from the above works in many ways including system model, objective function and performance metrics, and the derivations of our results are not based on those that have been known for stability problems. A further difference is our analysis for the problem with presence of deadlines.

For scheduling with deadlines, there has been a lot of work in wired networks or other interference-free systems (e.g., [16]-[18]), since the seminal work in [19], where the authors address the multiprogram scheduling in a hard-realtime environment. Scheduling with delay-constrained traffic or hard-real-time streams with deadlines have been studies in, for example, [20], [21]. However, the number of concurrently activated links (or streams) is known, whereas in our problem this is a part of the scheduling decision to be made.

\section{PRELIMINARIES}

Consider $N$ transmitter-receiver pairs, or links, denoted by $\mathcal{N}=\{1, \ldots, N\}$, and a strictly positive vector $\boldsymbol{d}=$ $\left(d_{1}, \ldots, d_{N}\right)^{T}$, in which $d_{i}$ is the finite amount of bit-traffic at the transmitter of link $i$. Let $\mathcal{H}$ denote the union of all nonempty subsets of $\mathcal{N}$, with $|\mathcal{H}|=2^{N}-1$. We use the term group to refer to a member $\mathcal{c} \in \mathcal{H}$. Note that $c$ itself is a set.

In general, there is a rate region for a given link group $c \in \mathcal{H}$, containing all the achievable rate vectors for the group. We use $\mathcal{S} \in \mathbb{R}_{+}^{N}$ to denote the overall rate region, that is, the collection of all rate vectors that we can choose to use. For convenience, we let $\mathcal{S}$ also include $\mathbf{0}$. For group $\mathcal{c}$, the rate region, denoted by $\mathcal{S}_{c}$, is the subset of $\mathcal{S}$ such that the links not in $\mathcal{C}$ have zero rates. Clearly, $\mathcal{S}=\left\{\cup_{\mathcal{c} \in \mathcal{H}} \mathcal{S}_{\mathcal{c}}, \mathbf{0}\right\}$. We do not impose specific structure requirements on $\mathcal{S}$ (which can be non-convex and possibly discrete), except for the following general properties that are natural for a wireless system with meaningful physical interpretation. First, $\mathcal{S}$ is a bounded and closed set, and the cardinality is finite in case $\mathcal{S}$ is discrete. Secondly, if any single link is transmitting by itself, its achievable rate is strictly positive. Third, since concurrently active links cause interference to each other, if some link is removed from a link group, the maximal achievable rates of the remaining links do not decrease (and possibly increase). That is, if $\boldsymbol{r}=\left(r_{1}, \ldots, r_{N}\right)^{T} \in \mathcal{S}$, then there is another rate vector $\boldsymbol{r}^{\prime}=\left(r_{1}^{\prime}, \ldots, r_{N}^{\prime}\right)^{T}$ such that $r_{i}^{\prime}=0$ and $r_{j}^{\prime} \geq r_{j}, \forall j \neq i$. We refer to this as the rate monotonicity property. As a result, if
$\mathcal{S}$ is convex, it is also coordinate-convex, i.e., if a rate vector $\boldsymbol{r}=\left(r_{1}, \ldots, r_{N}\right)^{T} \in \mathcal{S}$, then $\boldsymbol{r}^{\prime} \in \mathcal{S}, \forall \boldsymbol{r}^{\prime}: \boldsymbol{r} \geq \boldsymbol{r}^{\prime} \geq \mathbf{0}$. Moreover, for non-convex $\mathcal{S}$, we use $\mathcal{C}(\mathcal{S})$ to denote the convex hull of $\mathcal{S}$.

A special case of the above is when each group $\mathcal{c} \in \mathcal{H}$ is associated with one single rate vector $\boldsymbol{r}_{\mathcal{c}}$, i.e., the rate vector depends only on the composition of $c$. We refer to this scenario as the group-based rates (GBR). It is easy to see that for GBR, $\mathcal{S}$ is a discrete set.

For mathematical notation, throughout the paper, we use plain letters or numbers to denote scalars, and bold letters or numbers to denote vectors. Sets are denoted using calligraphic style.

\section{PROBlem Formulation}

The scheduling problem consists of determining the use of rate vectors (each associated with a link groups) and their activation durations. A mathematical formulation, at this stage, requires some caution, because $\mathcal{S}$ can be a set with infinitely many elements. In the following, we first restrict the scheduling formulation to using a finite set (but of arbitrary cardinality) of rate vectors from $\mathcal{S}$, and then prove that this, in fact, does not lead to loss of generality. Let us denote by $\boldsymbol{x}$ the decision vector of time durations of the rate vectors in a scheduling solution. To facilitate the derivation of our results, for the moment we use inequality for the data demand requirement.

$$
\begin{aligned}
T^{*}=\inf & \sum_{\boldsymbol{r} \in \tilde{\mathcal{S}}} x_{\boldsymbol{r}} \\
\text { s. t. } & \sum_{\boldsymbol{r} \in \tilde{\mathcal{S}}} \boldsymbol{r} x_{\boldsymbol{r}} \geq \boldsymbol{d} \\
& x_{\boldsymbol{r}} \geq 0, \boldsymbol{r} \in \tilde{\mathcal{S}}, \tilde{\mathcal{S}} \subseteq \mathcal{S}, \tilde{\mathcal{S}} \text { finite }
\end{aligned}
$$

The formulation above requires the use of a finite number of rate vectors. That is, $T^{*}$ is what can be obtained as infimum if the schedule is restricted to use a finite set of rate vectors. In the following, we will prove this restriction does not lead to loss of generality. Note that, the formulation is not a linear optimization problem. This is because even if $\tilde{\mathcal{S}}$ is restricted to be finite, the elements to be included are part of the optimization problem here. In general there are infinitely many choices of $\tilde{\mathcal{S}}$, and this is the reason of stating infimum, which exists as the schedule length is bounded from below by zero, rather than minimum in the formulation.

Lemma 1. The infimum in (1) cannot be improved by using an infinite number of rate vectors.

Proof: First, we observe that the rate vectors with positive time durations will not be uncountably infinite, because otherwise the schedule length becomes unbounded. Consider a schedule using countable but infinitely many rate vectors $\boldsymbol{r}_{1}, \boldsymbol{r}_{2}, \ldots$, with positive activation time durations $x_{\boldsymbol{r}_{1}}$, $x_{\boldsymbol{r}_{2}}, \ldots$, such that $\lim _{k \rightarrow \infty} \sum_{\ell=1}^{k} x_{\boldsymbol{r}_{\ell}}=\check{T}<T^{*}$, and $\lim _{k \rightarrow \infty} \sum_{\ell=1}^{k} \boldsymbol{r}_{\ell} x_{\boldsymbol{r}_{\ell}}=\boldsymbol{d}^{\prime} \geq \boldsymbol{d}$. Let $\lambda=T^{*} / \check{T}$. Since $\lambda>1$, there exists $k^{\prime}$, for which $\lambda \sum_{\ell=1}^{k^{\prime}} \boldsymbol{r}_{\ell} x_{\boldsymbol{r}_{\ell}} \geq \boldsymbol{d}$, as otherwise $\lim _{k \rightarrow \infty} \sum_{\ell=1}^{k} \boldsymbol{r}_{\ell} x_{\boldsymbol{r}_{\ell}}=\boldsymbol{d}^{\prime} \geq \boldsymbol{d}$ cannot hold. For 
these rate vectors, the corresponding time durations satisfy $\sum_{\ell=1}^{k^{\prime}} \lambda x_{\boldsymbol{r}_{\ell}}<\lambda \check{T}=T^{*}$, contradicting that $T^{*}$ is the infimum of the schedule length using any finite number of rate vectors. Hence $\check{T}=T^{*}$ and the lemma follows.

Lemma 2. The infimum $T^{*}$ defined in (1) is achievable and hence optimal, and the optimum is achieved with equality in (1b).

Proof: Consider activating one link at a time to deliver the demand vector, i.e., time division multiple access (TDMA). This gives obviously a feasible solution, of which the time length is denoted by $\hat{T}$. Because the objective is to have as short schedule as possible, adding an upper bound $x_{\boldsymbol{r}} \leq \hat{T}$ in (1c) does not cause loss of the generality of the formulation. The objective function (1a) is continuous on $x \in \mathcal{X}$, where $\mathcal{X}$ is the solution space defined by (1b) and the new (1c). Set $\mathcal{X}$ is closed, because this is the case for $\mathcal{S}$, and for the space defined by (1b) and (1c). Set $\mathcal{X}$ is also bounded, because of the boundedness of $\mathcal{S}$ and the upper bound $\hat{T}$ introduced to (1c). Hence, by the Weierstrass extreme value theorem, the infimum $T^{*}$ is also the optimum. A special case is discrete $\mathcal{S}$. As the number of rate vectors in $\mathcal{S}$ is finite in this case, the infimum is clearly the minimum.

For the equality in (1b), suppose the opposite, then there exists $\boldsymbol{r}$ with link $i$ 's rate $r_{i}>0$, run with time duration $x_{\boldsymbol{r}}$, such that for link $i$, the effective demand delivered by this rate vector, denoted by $d_{i \boldsymbol{r}}$, satisfies the condition $d_{i \boldsymbol{r}}<r_{i} x_{\boldsymbol{r}}$. Assuming that $\boldsymbol{r}$ is associated with group $c$ and letting $t=\frac{d_{i r}}{r_{i}}$, we split $x_{\boldsymbol{r}}$ into two segments, with lengths $t$ and $x_{\boldsymbol{r}}-t$, respectively. In the first segment, $\boldsymbol{r}$ is used, and for the second segment, a rate vector $\boldsymbol{r}^{\prime}$ for the reduced group $\mathcal{c} \backslash\{i\}$ is selected. By the rate monotonicity property, there exists at least one $\boldsymbol{r}^{\prime}$ where the links in $\mathcal{c} \backslash\{i\}$ have the same or better rates than in $\boldsymbol{r}$. This leads to a new solution that is able to deliver the same effective demand for all links in $c$, originally achieved by $r$ and time $x_{\boldsymbol{r}}$, using at most time $x_{\boldsymbol{r}}$, and the conclusion follows.

Thus, optimal scheduling in its general form can be formulated as follows.

$$
\begin{aligned}
T^{*}=\min & \sum_{\boldsymbol{r} \in \tilde{\mathcal{S}}} x_{\boldsymbol{r}} \\
\text { s. t. } & \sum_{\boldsymbol{r} \in \tilde{\mathcal{S}}} \boldsymbol{r} x_{\boldsymbol{r}}=\boldsymbol{d} \\
& x_{\boldsymbol{r}} \geq 0, \boldsymbol{r} \in \tilde{\mathcal{S}}, \tilde{\mathcal{S}} \subseteq \mathcal{S}, \tilde{\mathcal{S}} \text { finite }
\end{aligned}
$$

For scheduling with GBR, where each link group $\mathcal{c}$ is associated with a single rate vector $\boldsymbol{r}_{\mathcal{c}}$, the formulation can be made more specific as a linear program. For later reference, we provide the formulation below, where $x_{c}$ is the time duration of using group $c$ with rate vector $\boldsymbol{r}_{c}$, and $r_{i c}$ is the rate of link $i$ in group $c$. Note that the formulation (3) has an exponential size of $N$. In fact we have proved that scheduling with GBR remains hard with the continuous modeling of the traffic and the transmission time [13]. The underlying reason is that the scheduling problem's combinatorial nature is residing in selecting links to form transmission groups, and the rates that can be achieved follow from this discrete choice.

$$
\begin{array}{ll}
\min & \sum_{\mathcal{c} \in \mathcal{H}} x_{\mathcal{c}} \\
\text { s. t. } & \sum_{\mathcal{c} \in \mathcal{H}} r_{i c} x_{\mathcal{c}}=d_{i}, \forall i \in \mathcal{N} \\
& x_{\mathcal{c}} \geq 0, \mathcal{c} \in \mathcal{H}
\end{array}
$$

\section{OPTIMALITY CHARACTERIZATION}

Lemma 3. For (2), any feasible schedule, represented by the choice of rate set $\tilde{\mathcal{S}}$ and the values of the associated $x$ variables, is equivalent to a schedule using a single rate vector $\overline{\boldsymbol{r}}$, which is collinear with $\boldsymbol{d}$ and is a convex combination of $r \in \mathcal{S}$.

Proof: For any finite $\tilde{\mathcal{S}} \subseteq \mathcal{S}$, define scalar $T=\sum_{\boldsymbol{r} \in \tilde{S}} x_{\boldsymbol{r}}$ and $\lambda_{\boldsymbol{r}}=\frac{x_{\boldsymbol{r}}}{T}, \boldsymbol{r} \in \tilde{S}$. By definition, $\lambda_{\boldsymbol{r}}$ is the proportion of time using rate vector $r$. Formulation (2) can then be written as $\left\{\min T\right.$, s.t. $\left.T \sum_{\boldsymbol{r} \in \tilde{S}} \lambda_{\boldsymbol{r}} \boldsymbol{r}=\boldsymbol{d}, \quad \sum_{\boldsymbol{r} \in \tilde{S}} \lambda_{\boldsymbol{r}}=1, \boldsymbol{\lambda} \geq \mathbf{0}\right\}$. Let $\overline{\boldsymbol{r}}=\sum_{\boldsymbol{r} \in \tilde{S}} \lambda_{\boldsymbol{r}} \boldsymbol{r}$. By the first constraint, $T \overline{\boldsymbol{r}}=\boldsymbol{d}$, implying that $\overline{\boldsymbol{r}}$ is a scaling of $\boldsymbol{d}$, and rate vector $\overline{\boldsymbol{r}}$ meets the demand exactly with time $T$. By the next two constraints, $\overline{\boldsymbol{r}}$ is a convex combination of $\boldsymbol{r} \in \tilde{\mathcal{S}} \subseteq \mathcal{S}$, and the lemma follows.

Theorem 4. For any scheduling problem of (2), if $\mathcal{S}$ is convex, then it is optimal to use one rate vector $\boldsymbol{r}^{*}$ defined by $\boldsymbol{r}^{*} \in$ bd $\mathcal{S}$ (that is, $\boldsymbol{r}^{*}$ is a boundary point of $\mathcal{S}$ ) and $\boldsymbol{r}^{*}=\alpha \boldsymbol{d}, \alpha>$ 0 .

Proof: The problem (2) is clearly feasible. Moreover, because $\mathcal{S}$ is a bounded convex and coordinate-convex set in $\mathbb{R}_{+}^{N}$, given a sufficiently small scalar $\sigma>0$ and a sufficiently large scalar $\beta>0$, we have $\sigma \boldsymbol{d} \in \mathcal{S}$ and $\beta \boldsymbol{d} \notin \mathcal{S}$. This, together with the basic properties of convex sets, implies that the point $\boldsymbol{r}^{*}$, defined in the theorem, uniquely exists. For the optimality of $\boldsymbol{r}^{*}$, note first that for $\boldsymbol{r}^{*}$ the schedule length is $\frac{1}{\alpha}$. By Lemma 3, any feasible solution is equivalent to the use of one rate vector being collinear with $\boldsymbol{d}$, with a corresponding scaling value of, say $\alpha^{\prime}$, and hence schedule length $\frac{1}{\alpha^{\prime}}$. However, by construction, $\alpha^{\prime} \leq \alpha$. Hence $\boldsymbol{r}^{*}$ is optimal.

The corollary below directly follows from Theorem 4 for non-convex $\mathcal{S}$.

Corollary 5. For a scheduling problem with rate region $\mathcal{C}(\mathcal{S})$, using the single rate vector $\boldsymbol{r}^{*}$, wherein $\boldsymbol{r}^{*} \in \mathbf{b d} \mathcal{C}(\mathcal{S})$ and $\boldsymbol{r}^{*}=\alpha \boldsymbol{d}, \alpha>0$, is optimal.

In what follows, we denote by $\boldsymbol{r}^{*}(\mathcal{S})$ the rate vector corresponding to the intersection point of the ray defined by the demand vector $\boldsymbol{d}$ and the boundary of $\mathcal{C}(\mathcal{S})$.

Theorem 6. Optimal scheduling with arbitrary rate region $\mathcal{S}$ is equivalent to maximum scaling of the demand vector $d$ over the convex set $\mathcal{C}(\mathcal{S})$, that is, to solving the problem defined in (4) below, and the minimum schedule length equals $\frac{\|\boldsymbol{d}\| \|_{2}}{\left\|\boldsymbol{r}^{*}(\mathcal{S})\right\|_{2}}$.

Proof: By Carathéodory's theorem, any rate vector of $\mathcal{C}(\mathcal{S})$ can be represented as a convex combination of at most $N+1$ vectors of $\mathcal{S}$. Therefore, for any scheduling problem 
with a non-convex rate region $\mathcal{S}$, replacing $\mathcal{S}$ with its convex hull $\mathcal{C}(\mathcal{S})$ gives the same optimal value. This, together with Theorem 4 and Corollary 5, prove that the optimum of (2) is achieved by using rate vector $\boldsymbol{r}^{*}(\mathcal{S})$. This intersection point, by its definition, is the optimum to the following problem.

$$
\begin{array}{cl}
\max & \alpha \\
\text { s. t. } & \boldsymbol{r}=\alpha \boldsymbol{d} \\
& \alpha \geq 0, \boldsymbol{r} \in \mathcal{C}(\mathcal{S})
\end{array}
$$

Since $\boldsymbol{r}^{*}(\mathcal{S})$ and $\alpha^{*}$ are the optimal solution of (4), the optimal cost of (2) is calculated by $\frac{\|\boldsymbol{d}\|_{2}}{\left\|\boldsymbol{r}^{*}(\mathcal{S})\right\|_{2}}$, or equivalently, $\frac{1}{\alpha^{*}}$.

We illustrate the result in Figure 1 for $N=2$.

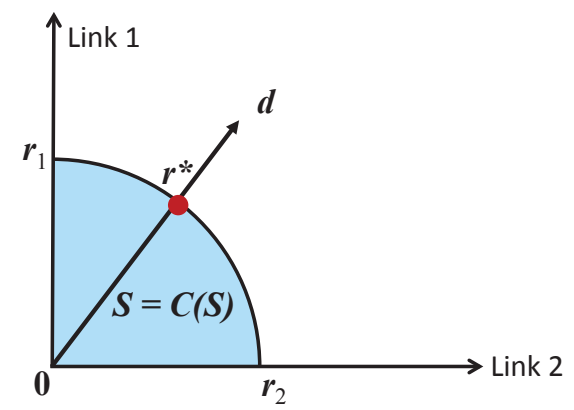

(a) Case 1: Convex rate region $\mathcal{S}$. The optimal solution uses a single rate vector $\boldsymbol{r}^{*}$.

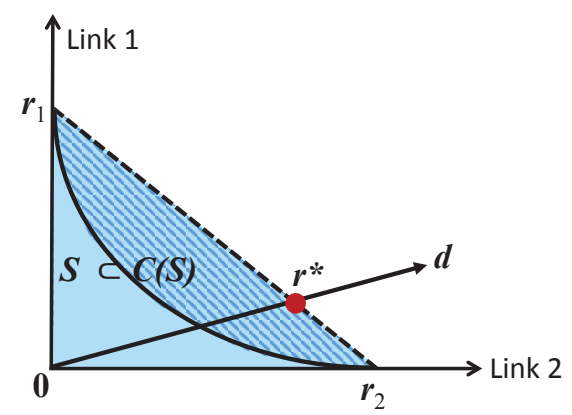

(b) Case 2: non-convex rate region $\mathcal{S}$, denoted by the non-shaded blue area. The optimum $\boldsymbol{r}^{*}$ is a convex combination of $\boldsymbol{r}_{1}$ and $\boldsymbol{r}_{2}$.

Fig. 1. Optimal scheduling with rate region $\mathcal{S}$ and $N=2$.

Remark 1. Although the objective function in (4) is maximization as opposite to that of (2), this formulation is not the same as the notion of duality in optimization. Moreover, for convex $\mathcal{S}$, solving (4) and obtaining $\alpha^{*}$ and $\boldsymbol{r}^{*}(\mathcal{S})$ are tractable. For non-convex $\mathcal{S}$, in general the optimal solution is achieved by a convex combination (i.e., time sharing) of up to $N+1$ vectors, see Figure $1 b$. It is in general hard to derive the convex combination for a non-convex $\mathcal{S}$, or, using $\mathcal{S}$ to find the optimal combination to construct $\boldsymbol{r}^{*}(\mathcal{S})$. Hence our result does not contradict the NP-hardness of the scheduling problem.

\section{EXAmples of Use of The Result}

The solution characterization in Theorem 6 is mathematically simple, yet powerful for unifying and extending the analysis of optimality and tractability of scheduling.

\section{A. Optimality Analysis of TDMA}

In words, Theorem 6 says that optimal scheduling is equivalent to maximum scaling of the demand vector without getting outside the convexified rate region. The result allows to study optimality of specific scheduling strategies, such as TDMA in which the demand requests of the links are satisfied by activating the respective links separately. That is, we investigate the condition under which the optimum $r^{*}(\mathcal{S})$ is a combination of the rates of the $N$ singleton groups $\mathcal{c}_{1}=\{1\}, \mathcal{c}_{2}=\{2\}, \ldots, \mathcal{c}_{N}=\{N\}$. Denote by $\mathcal{H}^{1}$ the TDMA schedule, and $r_{i i}$ the maximum achievable rate of link $i$ in $\mathcal{c}_{i}$. Since the links are activated fully separately, it is suboptimal to use any rate less than $r_{i i}$. Denote by $\boldsymbol{r}_{i}$ the rate vector in which the $i$ th element equals $r_{i i}$, and the rest are zeros. Define $\mathcal{C}\left(\mathcal{S}^{\prime}\right)=\mathcal{C}\left(\left\{\boldsymbol{r}_{1}, \ldots, \boldsymbol{r}_{N}, \mathbf{0}\right\}\right)$. For $\mathcal{C}\left(\mathcal{S}^{\prime}\right)$, which geometrically is an $N$-simplex, the solution, by Theorem 6 , is the intersection point of $\boldsymbol{d}$ and the surface of the simplex. If $\mathcal{C}(\mathcal{S}) \backslash \mathcal{C}\left(\mathcal{S}^{\prime}\right) \neq \emptyset$, then there is at least one rate vector beyond the simplex $\mathcal{C}\left(\mathcal{S}^{\prime}\right)$. As a result, $\mathcal{C}\left(\mathcal{S}^{\prime}\right) \subset \mathcal{C}(\mathcal{S})$ along the direction of any vector with strictly positive elements. Hence we obtain the following corollary.

Corollary 7. Schedule $\mathcal{H}^{1}$ is optimal, if and only if $\mathcal{C}(\mathcal{S})=$ $\mathcal{C}\left(\mathcal{S}^{\prime}\right)$.

Remark 2. The above condition is exact (i.e., both sufficient and necessary) and does not make any assumption on $\mathcal{S}$. Hence previous analyses, e.g., our result in [13] for scheduling with GBR using TDMA, are all special cases of Corollary 7.

\section{B. Minimum Weight Scheduling}

We use the solution characterization of Section V to study a generalized objective function, in which the cost per time unit differs by rate vector or group. That is, in the objective function, $x_{\boldsymbol{r}}$ is associated with a weight coefficient $\omega_{\boldsymbol{r}}, \omega_{\boldsymbol{r}}>$ 0 , and the problem reads $\left\{\min \sum_{\boldsymbol{r} \in \tilde{\mathcal{S}}} \omega_{\boldsymbol{r}} x_{\boldsymbol{r}}\right.$, s. t. $\left.(2 \mathrm{~b})-(2 \mathrm{c})\right\}$. Define $\overline{\boldsymbol{r}}=\frac{r}{\omega_{r}}, \boldsymbol{r} \in \mathcal{S}$, and let $\overline{\mathcal{S}}$ be the resulting rate region. Introducing variables $y_{\bar{r}}, \overline{\boldsymbol{r}} \in \overline{\mathcal{S}}$, we can then formulate the problem as follows.

$$
\begin{array}{ll}
\min & \sum_{\overline{\boldsymbol{r}} \in \tilde{\mathcal{S}}} y_{\overline{\boldsymbol{r}}} \\
\text { s. t. } & \sum_{\overline{\boldsymbol{r}} \in \tilde{\mathcal{S}}} \overline{y_{\overline{\boldsymbol{r}}}}=\boldsymbol{d} \\
& \boldsymbol{y} \geq \mathbf{0}, \overline{\boldsymbol{r}} \in \tilde{\mathcal{S}}, \tilde{\mathcal{S}} \subseteq \overline{\mathcal{S}}, \tilde{\mathcal{S}} \text { finite }
\end{array}
$$

Note that (5) has the same form as (2). Hence, structural properties and optimality of weighted scheduling can be studied, again using our results, and, in particular, Theorem 6. Here, we illustrate the case of minimum sum energy. Suppose we have the freedom to choose transmit power, such that each rate vector $\boldsymbol{r} \in \mathcal{S}$ is a result of a specific power vector (cf. power control). Consider any power vector $\left(p_{1}, \ldots, p_{N}\right)^{T}$ that leads to rate vector $r \in \mathcal{S}$, and denote its (strictly positive) sum power by $p_{\boldsymbol{r}}$. Thus $\omega_{\boldsymbol{r}}=p_{\boldsymbol{r}}$ and the corresponding, projected rate vector $\overline{\boldsymbol{r}}=\frac{\boldsymbol{r}}{p_{\boldsymbol{r}}}$. 
Consider activating one individual link $i$ using power $p_{i}$. We reuse notation $r_{i i}$, which has denoted the rate of link $i$ when this link is active alone, for the rate achieved by link $i$ with $p_{i}$. It is obvious that $r_{i i} \geq r_{i}$, where the latter is the rate element of $i$ in $\boldsymbol{r}$, because of the rate monotonicity property, which here means that if all links have the power values changed to zeros (or, equivalently, become inactive) except for one link, the rate of that link does not decrease. Thus we have the following inequality, where $\boldsymbol{r}_{i}$ denotes the rate vector in which the $i$ th element equals $r_{i i}$ and the other elements are zeros, and its projected vector $\overline{\boldsymbol{r}}_{i}$, by definition, equals $\frac{1}{p_{i}} \boldsymbol{r}_{i}$.

$$
\overline{\boldsymbol{r}}=\frac{1}{p_{\boldsymbol{r}}} \boldsymbol{r} \leq \sum_{i=1}^{N} \frac{1}{p_{\boldsymbol{r}}} \boldsymbol{r}_{i}=\sum_{i=1}^{N} \frac{p_{i}}{p_{\boldsymbol{r}}} \overline{\boldsymbol{r}}_{i}
$$

The last sum in (6) is a convex combination of $\overline{\boldsymbol{r}}_{i}, i=$ $1, \ldots, N$, which is a TDMA schedule. Hence any rate vector $\overline{\boldsymbol{r}} \in \overline{\mathcal{S}}$ is below the simplex defined by TDMA. As a result, the condition in Corollary 7 is fulfilled. Consequently TDMA is always energy-optimal.

Remark 3. It has become known that TDMA is optimal to achieve minimum energy (e.g., [22]), though under fixed power or some specific power-rate function. Our analysis is stronger, as it applies to arbitrary rate region and power-rate function.

\section{Tractability Consideration}

We leverage our results for problem tractability. By the ellipsoid method and its consequences in combinatorial optimization, a problem is polynomially solvable if and only if its separation problem has a polynomial-time solution [23]. By Theorem 6, finding the optimum indeed deals with separation, i.e., for a given point $\alpha \boldsymbol{d}$ with $\alpha>0$, to determine whether or not $\alpha \boldsymbol{d} \in \mathcal{C}(\mathcal{S})$, and the tractability of this task is the same as solving our scheduling problem to optimum.

The separation problem is in general hard for arbitrary $\mathcal{S}$. To identify tractable cases, we consider more specific rate region $\mathcal{S}$. In particular, we consider scheduling with GBR, for which $\mathcal{C}(\mathcal{S})$ is a polytope, and the separation is to determine if a point is within the polytope or not. For GBR, it has been proved that a special case of it, namely, the so called cardinality-based rates (CBR), is polynomial-time tractable [13]. In CBR, the transmission rate is determined completely by the cardinality of a group, and all links in a group share the same rate. An example scenario consists in the transmitters being in an isotropic medium at equal distances (on a circle) to the destination and hence with identical geometric gains to their common, or co-located, receivers at the center of the circle.

In the following, we consider a less restrictive problem class of which the tractability has not been revealed in previous studies. Consider the case where the maximum of link rate $r_{i c}$ is determined by the index $i$ and the group cardinality $|\boldsymbol{c}|$. Unlike CBR, the links of a group now have different rates, though the rate of a link is not dependent on the other elements of the group. We refer to this problem class as link-variable CBR. An example is given in Figure 2. From the illustration, we observe that, for any two vectors of the groups containing

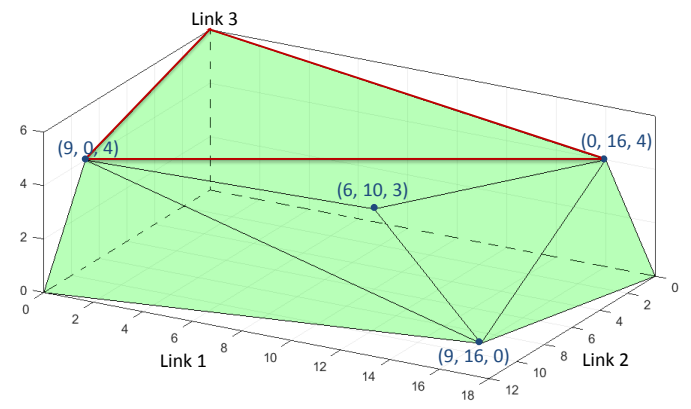

Fig. 2. An illustration of link-variable CBR for $N=3$.

two links, they have one rate value in common. As a result, for the specific values in the figure, to perform separation for any vector having a rate of at least 4 for link 3 , only one facet (as marked by the red edges) is relevant. This gives a hint that for this case, identifying the vertices that are relevant to the separation condition has a good chance of being tractable. Inspired by this, we formally prove the tractability result for link-variable CBR.

Theorem 8. Scheduling with link-variable CBR is polynomialtime solvable.

Proof: By the definition of link-specific CBR, we denote the rate of link $i$ in group $c$ as $r_{i n}$, wherein $n=|c|$. The LP dual of (3) for link-specific CBR is given below.

$$
\begin{array}{ll}
\max & \sum_{i \in \mathcal{N}} d_{i} \pi_{i} \\
\text { s. t. } & \sum_{i \in \mathcal{N}} r_{i n} \pi_{i} \leq 1 \quad \forall \mathcal{c} \in\{\mathcal{H}:|\boldsymbol{c}|=n\}, n=1, \ldots, N \\
& \boldsymbol{\pi} \geq \mathbf{0}
\end{array}
$$

The separation problem of (7) is to determine, for any given vector $\boldsymbol{\pi} \geq \mathbf{0}$, if it is in the feasible region defined by (7b). We observe that, for each $n$, there are $\left(\begin{array}{l}N \\ n\end{array}\right)$ constraints in (7b), each having at most $n$ non-zero terms in the left-hand side. Among these, the most stringent one is obviously that consisting of the $n$ largest elements in $\left\{r_{1 n} \pi_{1}, r_{2 n} \pi_{2}, \ldots, r_{N n} \pi_{N}\right\}$. Hence satisfying (7b) is equivalent to fulfilling these $N$ most stringent constraints. Consequently, the separation problem can be solved as follows. First, given the values of $r_{i n}$ and $\pi_{i}, i \in \mathcal{N}$ and $n=1, \ldots, N$, we calculate $r_{i n} \pi_{i}$ and sort them in descending order for each $n$. This step can be done with complexity $O\left(N^{2} \log N\right)$. Then, for each $n=1, \ldots, N$, we construct the most stringent constraint $\sum_{i \in \mathcal{N}} r_{i n} \pi_{i} \leq 1$ consisting of the top $n$ items of $r_{i n} \pi_{i}$ in the sorted sequence. Since there are in total $N$ constraints to be considered, solving the problem is polynomial in $N$. Hence the conclusion.

The problem class of link-variable CBR is of relevance to downlink in cellular scenarios with co-located transmitters and geometric-based gains. Here, $r_{i c}$ is determined by the signal strength of the link in question and how many links are active simultaneously. 


\section{SCHEDUling With DEADLINE FOR ONE LINK}

We now consider the significant extension of including time constraints, or deadlines. Herein, the deadline of a link refers to a time point by which the data of that link has to be completely delivered, counting from the starting point of the schedule. We first consider the case where only one link has deadline. To ease the presentation, we start with scheduling with GBR, defined in (3), and later extend the discussion to the general case defined in (2).

\section{A. Solution Characterization for $G B R$}

Without loss of generality, assume link one has deadline $T_{1}$. Denote by $\mathcal{H}_{i}$ the set of groups that link $i$ has positive rate. That is, $\mathcal{H}_{i}=\left\{c \in \mathcal{H}: r_{i c}>0\right\}$. With the presence of deadline, the order of groups in a schedule cannot be arbitrary. For the case of deadline for link one, however, this can be addressed by putting all chosen groups with link one first in the schedule. Hence link one is emptied in time $\sum_{\mathcal{c} \in \mathcal{H}_{1}} x_{\mathcal{c}}$.

Denote by $\check{\boldsymbol{x}}^{*}$ the optimal solution of (3), i.e., scheduling without deadline, and let $\check{T}_{1}^{*}=\sum_{c \in \mathcal{H}_{1}} \check{x}_{c}^{*}$. We assume that the deadline constraint of $T_{1}$ is non-redundant, i.e., $T_{1}<\check{T}_{1}^{*}$, as otherwise the optimum without any deadline would remain optimal. If $T_{1}<\frac{d_{1}}{r_{11}}$, where $r_{11}$ is the (maximum) rate of link one when it is activated alone, there is no feasible schedule. Hence we assume also $\frac{d_{1}}{r_{11}} \leq T_{1}$. The scheduling problem with one deadline can then be formulated as the following linear program that is (3) with an additional deadline constraint. Note that the equality of the demand constraint follows from the second part of the proof of Lemma 2.

$$
\begin{array}{ll}
\min & \sum_{c \in \mathcal{H}} x_{c} \\
\text { s. t. } & \sum_{c \in \mathcal{H}} r_{i c} x_{c}=d_{i} \quad i \in \mathcal{N} \\
& \sum_{c \in \mathcal{H}_{1}} x_{c}=T_{1} \\
& \boldsymbol{x} \geq \mathbf{0}
\end{array}
$$

We know that the scheduling problem without deadline is NP-hard [13]. Sometimes, introducing an additional constraint makes a hard optimization problem easier. This is however not the case here. In the following theorem we formally settle the problem's NP-hardness.

Theorem 9. The scheduling problem defined in (8) for which $T_{1}$ is non-redundant, i.e., the deadline alters the optimal schedule length of the problem without deadline, remains NPhard.

Proof: See the Appendix.

In Figure 3, we provide an illustration for $N=2$, for which there are three possible groups: $\mathcal{c}_{1}=\{1\}, \mathcal{c}_{2}=\{2\}$, and $\mathcal{c}_{3}=\{1,2\}$. By the results of Section $\mathrm{V}$, the purple point $\hat{\boldsymbol{r}}^{*}$, which maximizes demand scaling without leaving the polytope formed by $\boldsymbol{r}_{\mathcal{C}_{1}}, \boldsymbol{r}_{\mathcal{C}_{2}}, \boldsymbol{r}_{\mathcal{C}_{3}}$, and $\mathbf{0}$, is optimal without deadline, and this point amounts to using $\mathcal{c}_{1}=\{1\}$ and $\mathcal{c}_{3}=\{1,2\}$ with time sharing.

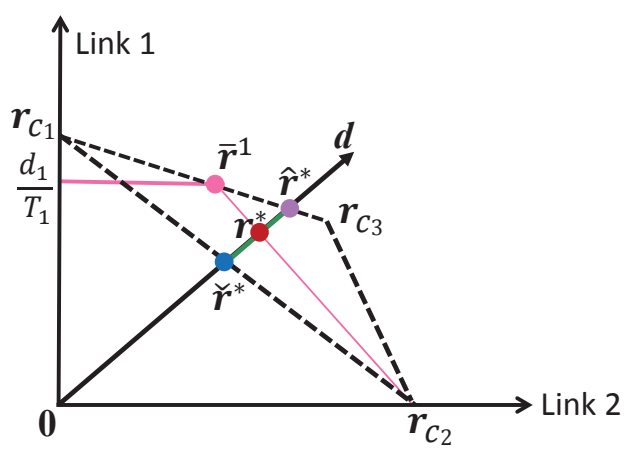

Fig. 3. The scheduling problem with one deadline and $N=2$.

Remark 4. For the example in Figure 3, for scheduling without deadline, all feasible schedules are on the green line segment, defined by $\check{\boldsymbol{r}}^{*}$ and $\hat{\boldsymbol{r}}^{*}$, and the latter gives the maximum demand vector scaling or equivalently shortest schedule. A point on the line below $\check{\boldsymbol{r}}^{*}$ is artificially feasible, if the schedule has an idle time period (i.e., no group activation), as such a point involves the zero vector for time sharing, as seen in the figure. This is clearly not optimal and hence such solutions are not considered here.

With the deadline $T_{1}$, the total activation time of all groups in $\mathcal{H}_{1}=\left\{c_{1}, \mathcal{c}_{3}\right\}$, must be no longer than $T_{1}$. This is equivalent to stating that the average rate of link one, achieved via activating groups $\mathcal{c}_{1}$ and $\mathcal{c}_{3}$, is at least $\frac{d_{1}}{T_{1}}$. In other words, the time sharing of these two specific groups (which appear first in the schedule) must be such, that the resulting rate of link one is $\frac{d_{1}}{T_{1}}$. This exactly maps to the segment between $\boldsymbol{r}_{c 1}$ and $\overline{\boldsymbol{r}}^{1}$. Hence, the rate region that is feasible with respect to the deadline is defined by $\left\{\boldsymbol{r}_{c 1}, \overline{\boldsymbol{r}}^{1}, \boldsymbol{r}_{c 2}, \mathbf{0}\right\}$. Next, note that Lemma 3 is not affected by the deadline. Hence the scheduling solution corresponds to a single vector being collinear with $\boldsymbol{d}$. Also, the objective remains to maximize the scaling of $\boldsymbol{d}$. Hence it becomes evident that the optimum with deadline for link one is $\boldsymbol{r}^{*}$, marked red. As shown by the figure, solution $\boldsymbol{r}^{*}$ is achieved by activating all three groups with time sharing.

The example indicates that the deadline constraint can be naturally "absorbed" into the definition of rate region of relevance. We formalize this result below. Define rate vector $\boldsymbol{r}^{1}(\boldsymbol{x})=\frac{\sum_{c \in \mathcal{H}_{1}} x_{c} \boldsymbol{r}_{c}}{\sum_{c \in \mathcal{H}_{1}} x_{c}}$, which is a convex combination of $\boldsymbol{r}_{c}$, $\mathcal{c} \in \mathcal{H}_{1}$, in a solution $\boldsymbol{x}$.

Lemma 10. A solution $\boldsymbol{x}$ of the scheduling problem (3) is feasible to (8) if and only if the first element of $\boldsymbol{r}^{1}(\boldsymbol{x})$, i.e., $r_{1}^{1}(\boldsymbol{x})$, equals $\frac{d_{1}}{T_{1}}$.

Proof: By the definition of $\boldsymbol{r}^{1}(\boldsymbol{x})$, we have $\bar{r}_{1}^{1}(\boldsymbol{x})=$ $\frac{\sum_{c \in \mathcal{H}_{1}} x_{c} r_{1 c}}{\sum_{c \in \mathcal{H}_{1}} x_{c}}$, which is the average rate of link one induced by $\boldsymbol{x}$. As the deadline constraint must be binding in (8), $r_{1}^{1}(\boldsymbol{x})=\frac{d_{1}}{T_{1}}$. For sufficiency, if $r_{1}^{1}(\boldsymbol{x})=\frac{d_{1}}{T_{1}}$, then the deadline is met because the active groups in $\mathcal{c} \in \mathcal{H}_{1}$ are at the very front the schedule.

We now formalize the corresponding result of Theorem 6 for the scheduling with one link deadline. Note that for GBR, as formulated in (8), the rate region $\mathcal{S}=\left\{\boldsymbol{r}_{\mathcal{c}}: \mathcal{c} \in \mathcal{H}, \mathbf{0}\right\}$, 
where $\boldsymbol{r}_{c}$ is the rate vector of group $c \in \mathcal{H}$. Clearly, $\mathcal{C}(\mathcal{S})$ is a polytope.

Theorem 11. For the scheduling problem with one deadline, as defined in (8), the optimal solution is equivalent to using one single rate vector $\boldsymbol{r}^{*}$ defined by $\boldsymbol{r}^{*}=\alpha \boldsymbol{d}, \alpha>0$, and $r^{*} \in \operatorname{bd} \mathcal{C}\left(\mathcal{S}^{\prime}\right)$, wherein $\mathcal{C}\left(\mathcal{S}^{\prime}\right)$ is the polytope of which the vertices are in $\mathcal{S}^{\prime}=\left\{\boldsymbol{r}_{c}: \mathcal{C} \in \mathcal{H} \backslash \mathcal{H}_{1}, \mathbf{0}\right\} \cup\left\{\mathbf{e} \mathcal{C}(\mathcal{S}): r_{1}=\right.$ $\frac{d_{1}}{T_{1}}$, where e $\mathcal{C}(\mathcal{S})$ denotes the edges of polytope $\mathcal{C}(\mathcal{S})$.

Proof: Any solution $\boldsymbol{x}$ of (3), by Corollary 5, corresponds to a single rate vector of $\mathcal{C}(\mathcal{S})$, and this vector is a convex combination of $\boldsymbol{r}_{c}, \mathcal{c} \in \mathcal{H}$. By the definitions of $\mathcal{H}_{1}$ and $\boldsymbol{r}^{1}(\boldsymbol{x})$, the vector is equivalently a convex combination of $\boldsymbol{r}^{1}(\boldsymbol{x})$ and $\left\{\boldsymbol{r}_{c}, \mathcal{c} \in \mathcal{H} \backslash \mathcal{H}_{1}\right\}$. By Lemma 10, $\boldsymbol{x}$ is feasible to (8) if and only if $r_{1}^{1}(\boldsymbol{x})=\frac{d_{1}}{T_{1}}$. Hence the solution is a convex combination of rate vectors of $\left\{\boldsymbol{r} \in \mathcal{C}(\mathcal{S}): r_{1}=\frac{d_{1}}{T_{1}}\right\}$ and $\left\{\boldsymbol{r}_{\boldsymbol{c}}: \quad \boldsymbol{c} \in \mathcal{H} \backslash \mathcal{H}_{1}\right\}$. For the former, any element can be represented as a convex combination of a finite number of rate vectors in $\left\{\mathbf{e} \mathcal{C}(\mathcal{S}): r_{1}=\frac{d_{1}}{T_{1}}\right\}$, because $\mathcal{C}(\mathcal{S})$ is a polytope. Therefore, a solution to (8) is a convex combination of elements in set $\mathcal{S}^{\prime}$ that defined in the theorem. Consequently, (8) is equivalent to (3) with rate region $\mathcal{C}\left(\mathcal{S}^{\prime}\right)$. By the result in Theorem 6 , the optimal solution corresponds to using one single rate vector $\boldsymbol{r}^{*}$ defined by $\boldsymbol{r}^{*}=\alpha \boldsymbol{d}, \alpha>0$, and $\boldsymbol{r}^{*} \in \operatorname{bd} \mathcal{C}\left(\mathcal{S}^{\prime}\right)$.

\section{B. Extension to Arbitrary Rate Region}

Consider now the general case of rate region $\mathcal{S}$, and denote by $\mathcal{S}_{i}$ the sub-region for which link $i$ has strictly positive rate. That is, $\mathcal{S}_{i}=\left\{\boldsymbol{r} \in \mathcal{S}: r_{i}>0\right\}$. The problem formulation, as shown below, is (2) plus the deadline constraint for link one.

$$
\begin{array}{ll}
\min & \sum_{\boldsymbol{r} \in \tilde{\mathcal{S}}} x_{\boldsymbol{r}} \\
\text { s. t. } & \sum_{\boldsymbol{r} \in \tilde{\mathcal{S}}} \boldsymbol{r} x_{\boldsymbol{r}}=\boldsymbol{d} \\
& \sum_{\boldsymbol{r} \in \tilde{\mathcal{S}}_{1}} x_{\boldsymbol{r}}=T_{1} \\
& \boldsymbol{x} \geq \mathbf{0}, \boldsymbol{r} \in \tilde{\mathcal{S}}, \tilde{\mathcal{S}} \subseteq \mathcal{S}, \tilde{\mathcal{S}}_{1} \subseteq \mathcal{S}_{1}, \tilde{\mathcal{S}}, \tilde{\mathcal{S}}_{1} \text { finite }
\end{array}
$$

Theorem 12. For the scheduling problem defined in (9), the optimal solution is equivalent to using one single rate vector $\boldsymbol{r}^{*}$ defined by $\boldsymbol{r}^{*}=\alpha \boldsymbol{d}, \alpha>0$, and $\boldsymbol{r}^{*} \in \operatorname{bd} \mathcal{C}\left(\mathcal{S}^{\prime}\right)$, wherein $\mathcal{C}\left(\mathcal{S}^{\prime}\right)$ is the convex hull of $\mathcal{S}^{\prime}=\left\{\boldsymbol{r} \in \mathcal{S} \backslash \mathcal{S}_{1}\right\} \cup$ $\left\{\operatorname{bd} \mathcal{C}(\mathcal{S}): r_{1}=\frac{d_{1}}{T_{1}}\right\}$

Proof: The proof is easily obtained by following the line of arguments in the proofs of Lemma 10 and Theorem 11, and adapting them to a general rate region $\mathcal{S}$.

\section{Geometric Interpretation and Illustrations}

We provide additional illustrations for GBR and $N=3$ links, to further shed light on the connection between deadline and rate region. In Figure 4(a), the green polytope is $\mathcal{C}(\mathcal{S})$. By the previous analysis, the optimal solution is $\hat{r}^{*}$, achieved by time sharing (i.e., a convex combination) of $\boldsymbol{r}_{1}, \boldsymbol{r}_{4}$, and $\boldsymbol{r}_{7}$.
With the presence of deadline for link one, the edge points of the polytope for which the rate of link one equals $\frac{d_{1}}{T_{1}}$ are $\left\{\boldsymbol{r}_{8}, \boldsymbol{r}_{9}, \boldsymbol{r}_{10}, \boldsymbol{r}_{11}\right\}$. By Theorem 11, the new rate region incorporating the deadline constraint is the polytope of which the vertices are these points plus those in the original polytope for which the rate of link one is zero. The latter consists of $\left\{\boldsymbol{0}, \boldsymbol{r}_{2}, \boldsymbol{r}_{3}, \boldsymbol{r}_{5}\right\}$. The resulting polytope is shown in Figure 4(b). Optimizing the schedule with this polytope (without deadline constraint) is equivalent to considering the deadline of link one. The optimal solution is the red point $\boldsymbol{r}^{*}$, which is a convex combination of rate vectors $\boldsymbol{r}_{2}, \boldsymbol{r}_{5}$, and $\boldsymbol{r}_{11}$ which in turn is a convex combination of $\boldsymbol{r}_{1}$ and $\boldsymbol{r}_{7}$.

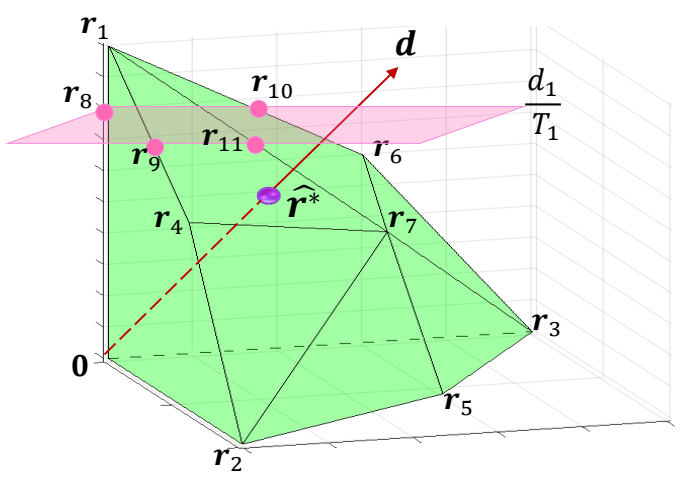

(a)

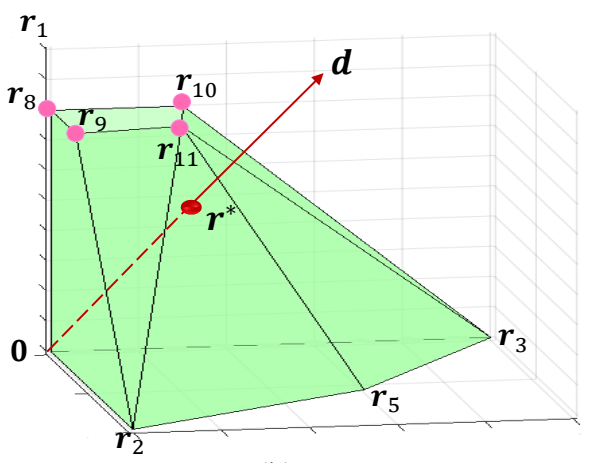

(b)

Fig. 4. Geometric illustration of optimal scheduling with deadline for GBR.

Note that the new polytope is not what remains by simply cutting the original one with a horizontal plane (see Figure 4(a)). For the example, the shape below the plane has changed as the vertices $\boldsymbol{r}_{4}, \boldsymbol{r}_{6}$, and $\boldsymbol{r}_{7}$ are no longer part of the new polytope.

For the general case of scheduling with rate region $\mathcal{S}$, we provide an illustration of Theorem 12 in Figure 5. In Figure 5(a), the green solid (including the entire interior), represents the convexified rate region $\mathcal{C}(\mathcal{S})$. The demand vector is shown by the dashed line. By the analysis of Section $\mathrm{V}$, the optimum without having deadline is the purple point $\hat{\boldsymbol{r}}^{*}$. To address the deadline constraint by adapting the rate region, we consider the intersection of the pink cutting plane $r_{1}=\frac{d_{1}}{T_{1}}$ and the boundary of $\mathcal{C}(\mathcal{S})$, i.e., $\left\{\mathbf{b d} \mathcal{C}(\mathcal{S}): r_{1}=\frac{d_{1}}{T_{1}}\right\}$, seen as the boundaries of the quarter-circle in Figure 5(a). By Theorem 12, the new rate region is then the convex hull of these points and $\left\{\boldsymbol{r} \in \mathcal{S}: r_{1}=0\right\}$, as shown by the solid in Figure 5(b). Optimal scheduling with deadline is equivalent to using $\boldsymbol{r}^{*}$. 


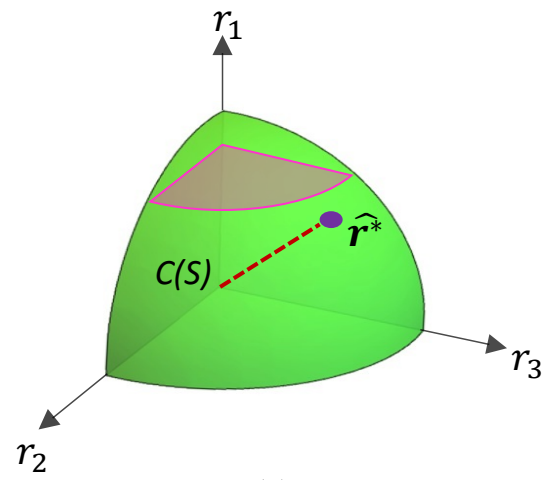

(a)

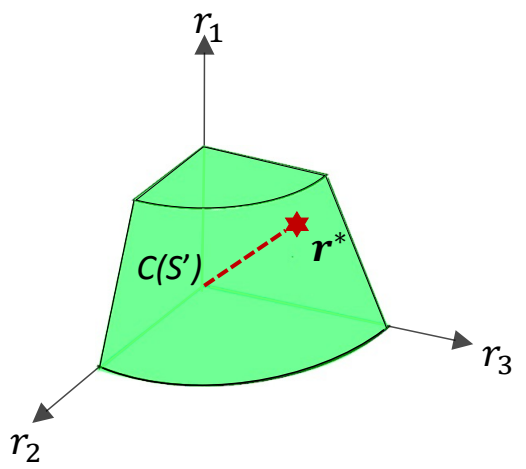

(b)

Fig. 5. Geometric illustration of optimal scheduling with deadline for general rate region.

As for the previous illustration, note again that the new rate region is not the part of the original one below $r_{1}=$ $\frac{d_{1}}{T_{1}}$. In particular, the two curves on the sides of the solid in Figure 5(a) become straight line segments in the new rate region, and, for this reason, $\left\|\boldsymbol{r}^{*}\right\|_{2}<\left\|\hat{\boldsymbol{r}}^{*}\right\|_{2}$.

\section{Scheduling With Deadlines For Multiple LINKS}

We consider optimal scheduling where multiple and possibly all links have deadlines. Denote the deadlines by $T_{i}, i=$ $1, \ldots, N$, and assume that $T_{1} \leq T_{2} \leq \cdots \leq T_{N}$. A schedule is feasible only if the demand of link $i$ is fully delivered by time $T_{i}$. We remark that, if there exists any feasible solution, then deadline $T_{N}$ is redundant, because this is in fact the overall schedule length, which is to be minimized. Hence we will not explicitly address $T_{N}$.

\section{A. Problem Formulation}

The order of selected link groups is not obvious at all for scheduling with multiple deadlines, since the completion times of the links are intertwined via the selection and activation times of the groups. Note that all previous formulations use group activation times (represented by $\boldsymbol{x}$ ), which by themselves do not tell the activation order. We will show how to formulate the deadline constraints without introducing additional variables, thus enabling to extend our previous analysis to multiple deadlines. In particular, in Theorem 13, we prove an important property, stating that to determine if a solution given by $\boldsymbol{x}$ can meet all the deadline constraints, it is sufficient and necessary to examine $\boldsymbol{x}$ with one specific partial ordering of its individual active groups.

Given $\boldsymbol{x}$, we construct (at most) $N$ segments as follows. The first segment contains all active groups with link one. The second segment includes all the groups that has link two, except those in which both links one and two are present, and so on, such that the $i$ th segment are the groups in $\boldsymbol{x}$ containing link $i$ but not any of the links in $\{1, \ldots, i-1\}$. The order of groups in each segment can be arbitrary. For any group, we refer to the earliest deadline of all links of the group as the group deadline. In general, any ordering in ascending order of group deadlines is referred to as earliest-deadline-group-first $(E D G F)$. We remark that the above construction of segments for any given $\boldsymbol{x}$ follows the EDGF order.

Theorem 13. Given $\boldsymbol{x}$, its active groups can be ordered to meet all the deadlines if and only if this is the case for EDGF.

Proof: The sufficiency follows directly from that EDGF is a scheduling solution. For necessity, assume there exists some ordering of the active groups of $\boldsymbol{x}$, such that all the deadlines are met. Suppose this sequence does not follow EDGF. Then in the sequence, some group $c_{n}$ containing link $n$ but not any link in $\{1, \ldots, i\}$, is scheduled before a group $c_{i}$ that contains link $i$, with $i<n$. Since this sequence meets all the deadlines, the deadline of link $i$, i.e., $T_{i}$, has not expired by the end of the activation time of $\mathcal{c}_{i}$. Consider moving group $\mathcal{c}_{n}$ to be immediately after $\mathcal{c}_{i}$ instead. Group $\mathcal{c}_{i}$, as well as the groups activating in between $\mathcal{c}_{n}$ and $\mathcal{c}_{i}$, are shifted earlier in time. Group $\mathcal{c}_{n}$ is still scheduled before $T_{n}$ because $T_{i} \leq T_{n}$. The rest of the groups after $c_{i}$ in the sequence remain unchanged. Therefore the new sequence meets the deadlines as well. It is apparent that after repeating the process in a finite number of steps, the sequence will follow EDGF, hence the conclusion.

From now on, we assume that EDGF applies to any schedule solution $\boldsymbol{x}$ under consideration. This leads to the following formulation (10) that does not require additional variables than $\boldsymbol{x}$.

$$
\begin{array}{ll}
\min & \sum_{\boldsymbol{r} \in \tilde{\mathcal{S}}} x_{\boldsymbol{r}} \\
\text { s. t. } & \sum_{\boldsymbol{r} \in \tilde{\mathcal{S}}} \boldsymbol{r} x_{\boldsymbol{r}}=\boldsymbol{d} \\
& \sum_{\boldsymbol{r} \in \tilde{\mathcal{S}}_{1} \cup \tilde{\mathcal{S}}_{2} \cup \ldots \cup \tilde{\mathcal{S}}_{n}} x_{\boldsymbol{r}} \leq T_{n} \quad n=1,2, \ldots, N-1 \\
& \boldsymbol{x} \geq \mathbf{0}, \boldsymbol{r} \in \tilde{\mathcal{S}}, \tilde{\mathcal{S}} \subseteq \mathcal{S}, \tilde{\mathcal{S}}_{n} \subseteq \mathcal{S}_{n}, \tilde{\mathcal{S}}, \tilde{\mathcal{S}}_{n} \text { finite, } \\
& n=1,2, \ldots, N-1
\end{array}
$$

\section{B. Solution Characterization}

To reach an optimality characterization to (10), the idea, similar as before, amounts to converting the deadline constraints defined in terms of "time" to "rate". For a scheduling solution $\boldsymbol{x}$, suppose $\overline{\mathcal{S}}_{1}, \overline{\mathcal{S}}_{2}, \ldots \overline{\mathcal{S}}_{n}$ are the respective sets of rate vectors in $\mathcal{S}_{1}, \mathcal{S}_{2}, \ldots, \mathcal{S}_{n}$ used by $\boldsymbol{x}$. Define $\boldsymbol{r}^{n}(\boldsymbol{x})=$ 


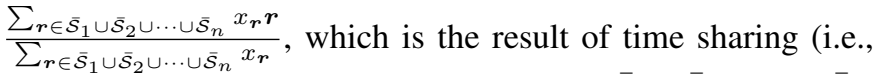
convex combination) of the rate vectors in $\overline{\mathcal{S}}_{1} \cup \overline{\mathcal{S}}_{2} \cup \cdots \cup \overline{\mathcal{S}}_{n}$. Element $r_{n}^{n}(\boldsymbol{x})$ is the average rate achieved by $\boldsymbol{x}$ for link $n$, at the time point when the activation of all groups in $\overline{\mathcal{S}}_{1}, \overline{\mathcal{S}}_{2}, \ldots \overline{\mathcal{S}}_{n}$ are scheduled, following the order of EDGF. Because there is no group later in the schedule containing $n$, the $n$th constraint in (10c) is satisfied by $\boldsymbol{x}$ if and only if $r_{n}^{n}(\boldsymbol{x}) \geq \frac{d_{n}}{T_{n}}$, for $n=1, \ldots, N-1$.

By the above rationale, we iteratively leverage the result in Section VII-B. Specifically, starting from $\mathcal{C}(\mathcal{S})$, we revise the rate region in each step, such that minimum-time scheduling over the new region is equivalent to that over the original rate region $\mathcal{C}(\mathcal{S})$ with the deadline constraint. Define $\mathcal{S}^{\prime(0)}=\mathcal{S}$, and, in the $n$th step, denote by $\mathcal{C}\left(\mathcal{S}^{\prime(n)}\right)$ the convex hull of $\mathcal{S}^{\prime(n)}=\left\{\operatorname{bd} \mathcal{C}\left(\mathcal{S}^{\prime(n-1)}\right): r_{n} \geq \frac{d_{n}}{T_{n}}\right\} \cup\left(\mathcal{S} \backslash\left(\mathcal{S}_{1} \cup \mathcal{S}_{2} \cdots \cup\right.\right.$ $\left.\mathcal{S}_{n}\right)$ ). Here, $\mathcal{S}^{\prime(n)}$ is the revised rate region after incorporating deadlines of the first $n$ links. This, in term, has two parts. The first part is the boundary of the corresponding rate region for up to first $n-1$ links with the constraint $r_{n} \geq \frac{d_{n}}{T_{n}}$ to account for the deadline of link $n$, and the second part is all rate vectors achieved using only links $n+1, \ldots, N$, as the deadlines of these links are not considered yet in this iteration.

Theorem 14. For (10), the optimal solution is equivalent to using one single rate vector $\boldsymbol{r}^{*}$ defined by $\boldsymbol{r}^{*}=\alpha \boldsymbol{d}, \alpha>$ 0 , and $\boldsymbol{r}^{*} \in \operatorname{bd} \mathcal{C}\left(\mathcal{S}^{\prime(N-1)}\right)$, with $\mathcal{S}^{\prime(0)}=\mathcal{S}$ and $\mathcal{S}^{\prime(n)}=$ $\left\{\right.$ bd $\left.\mathcal{C}\left(\mathcal{S}^{\prime(n-1)}\right): r_{n} \geq \frac{d_{n}}{T_{n}}\right\} \cup\left(\mathcal{S} \backslash\left(\mathcal{S}_{1} \cup \mathcal{S}_{2} \cdots \cup \mathcal{S}_{n}\right)\right)$, $n=1,2, \ldots, N-1$.

Proof: Applying Theorem 6 to rate region $\mathcal{S}^{\prime(N-1)}, \boldsymbol{r}^{*}$, as defined above, exists and is the optimal scheduling rate vector for $\mathcal{S}^{\prime(N-1)}$. We prove that $\boldsymbol{r}^{*}$ is feasible to (10). By definition, $\boldsymbol{r}^{*} \in \mathcal{C}\left(\mathcal{S}^{\prime(N-1)}\right) \subseteq \mathcal{C}\left(\mathcal{S}^{\prime(N-2)}\right) \subseteq \cdots \subseteq \mathcal{C}\left(S^{\prime(1)}\right) \subseteq \mathcal{C}(\mathcal{S})$. Hence, for each $n=1,2, \ldots, N-1, \boldsymbol{r}^{*}$ can be broken down into a convex combination of two groups of rate vectors in $\mathcal{S}_{1} \cup \mathcal{S}_{2} \cup \cdots \cup \mathcal{S}_{n}$ and $\mathcal{S} \backslash\left(\mathcal{S}_{1} \cup \mathcal{S}_{2} \cup \cdots \cup \mathcal{S}_{n}\right)$, respectively. This is because the two parts are mutually exclusive and together form $\mathcal{S}$. Denote by $\boldsymbol{r}^{*(n)}$ the convex combination of the vectors of the former group. By the definition of $\mathcal{S}^{\prime(n)}$, $\boldsymbol{r}^{*(n)} \in \mathcal{S}^{\prime(n)}$. Moreover, the rate vectors of the latter group have no impact on the completion time for link $n$, because of EDGF. By the construction of the rate region sequence, $\boldsymbol{r}^{*(n)}$ can be represented as a convex combination of rate vectors in $\left\{\operatorname{bd} \mathcal{C}\left(\mathcal{S}^{\prime(n-1)}\right): r_{n} \geq \frac{d_{n}}{T_{n}}\right\}$, of which the $n$th elements are all at least $\frac{d_{n}}{T_{n}}$. As a result, $r_{n}^{*(n)}$ is $\frac{d_{n}}{T_{n}}$ by minimum. This is the average rate of link $n$, from the beginning of the schedule until the time point when the activation of all link groups containing any of the links $1, \ldots, n$ are completed, following the order of EDGF. Hence the $n$th constraint in (10c) is satisfied. Applying this argument to $n=1,2, \ldots, N-1, \boldsymbol{r}^{*}$ corresponds to a feasible solution of (10).

Next, we show that any feasible solution $\boldsymbol{x}$ of (10) corresponds to a rate vector $\overline{\boldsymbol{r}} \in \mathcal{C}\left(\mathcal{S}^{\prime(N-1)}\right)$. Because $\boldsymbol{x}$ is also a feasible solution for scheduling without deadline but with rate region $\mathcal{S}$, by Lemma 3 , it is equivalent to using some rate vector $\overline{\boldsymbol{r}} \in \mathcal{C}(\mathcal{S})$. Vector $\overline{\boldsymbol{r}}$ can be broken down into a convex combination of a rate vector $\overline{\boldsymbol{r}}^{(1)} \in \mathcal{C}\left(\mathcal{S}_{1}\right)$ and a second rate vector in $\mathcal{C}\left(\mathcal{S} \backslash \mathcal{S}_{1}\right)$. Because $\overline{\boldsymbol{r}}$ meets the deadline of link one, $\bar{r}_{1}^{(1)} \geq \frac{d_{1}}{T_{1}}$, due to EDGF and the fact that link one does not appear in $\mathcal{S} \backslash \mathcal{S}_{1}$. Hence $\overline{\boldsymbol{r}}^{(1)} \in\left\{\mathbf{b d} \mathcal{C}\left(\mathcal{S}^{\prime(0)}\right): r_{1} \geq \frac{d_{1}}{T_{1}}\right\}$. As a result, $\overline{\boldsymbol{r}} \in \mathcal{S}^{\prime(1)}$. Consider $n=2$. Rate vector $\overline{\boldsymbol{r}}$ can be broken down into a vector $\overline{\boldsymbol{r}}^{(2)} \in \mathcal{C}\left(\mathcal{S}_{1} \cup \mathcal{S}_{2}\right)$ and a second vector in $\mathcal{C}\left(\mathcal{S} \backslash\left(\mathcal{S}_{1} \cup \mathcal{S}_{2}\right)\right)$. Because $\overline{\boldsymbol{r}}$ satisfies the deadline of link two, $\bar{r}_{2}^{(2)} \geq \frac{d_{2}}{T_{2}}$, again because of EDGF and the that activation of link two does not happen in $\mathcal{S} \backslash\left(\mathcal{S}_{1} \cup \mathcal{S}_{2}\right)$. This, together with $\overline{\boldsymbol{r}} \in \mathcal{S}^{\prime(1)}$, establish the fact that $\overline{\boldsymbol{r}} \in \mathcal{S}^{\prime(2)}$. Repeating the argument for $n \geq 2, \bar{r} \in \mathcal{C}\left(\mathcal{S}^{\prime(N-1)}\right)$. Hence, (10) is equivalent to (2) with rate region $\mathcal{C}\left(\mathcal{S}^{\prime(N-1)}\right)$, and the theorem follows.

For scheduling without deadline, at most $N+1$ rate vectors are needed to define the optimum. This remains true with deadlines, as deadlines are equivalent to revising the rate region.

Corollary 15. For (10), there exists an optimal solution $\boldsymbol{r}^{*}$ which is the result of time sharing of at most $N+1$ rates in $\mathcal{S}$.

\section{CONCLUSIONS}

Optimal link scheduling has been among the first problems studied in depth. Yet the problem continues to unfold and reveal its multiple facets as we formulate and analyze it. In the paper, we have provided optimality characterization for the scheduling problem and in its most general form. This turned out to be very useful to unify and to extend previous results in a variety of aspects. Moreover, leveraging our approach enables to understand the effect of deadlines, by proving how these time constraints can be equivalently formulated from the rate region viewpoint, thus demonstrating the strength of the analytical framework. Based on the results, there are a number of possible extensions for further study, including recognition of tractable problem classes with presence of deadline and the analysis of dynamic scenarios with arrivals.

\section{APPENDIX}

Proof of NP-HARDNESS OF THE SCHEDULING PROBLEM WITH DEADLINE

Proof: We prove the problem of (8) is NP-hard by a polynomial-time reduction from the scheduling problem defined in (3), which has been proved to be NP-hard in [13]. Before proceeding to the proof itself, it should be remarked that, as long as complexity is concerned, we do not consider the link rates of the $2^{N}-1$ groups as part of the problem input. For complexity analysis, the input size is defined by $N$, and formulations (3) and (8) serve the purpose of formalize the respective problems, instead of LPs that are explicitly given. For problem solving, whenever the rate vector of a link group is needed for optimization, an algorithm uses some function to compute the vector. The algorithm is of polynomial-time complexity, if the number of computations and the number of function evaluations required are both polynomial in $N$. Henceforth, we use $f$ to denote the rate function for (3), i.e., $f(c)=\boldsymbol{r}_{c}, c \in \mathcal{H}$.

Given $N$ links and function $f$, we reduce any problem instance without deadline to an instance with one deadline as follows. We introduce two additional links $N+1$ and $N+2$, 
both having a demand of two. For the singleton groups $\{N+1\}$ and $\{N+2\}$, the rate function $f$ returns rate 3 . For group $\{N+1, N+2\}$, the rate values of the two links are $(2,2)^{T}$. Moreover, for any group that jointly contains $N+1$ or $N+2$ along with at least one link in $\mathcal{N}$, the rate function returns the zero vector, that is, $f(\boldsymbol{c})=\mathbf{0}$ if $\mathcal{c} \cap\{N+1, N+2\} \cap \mathcal{N} \neq \emptyset$. The rate computed by $f$ remains for the instance of $\mathcal{N}$ links, for any link group $c \in \mathcal{H}$.

For the constructed instance, it is easy to see that the optimal schedule will not use any group $c$ such that $c \cap\{N+1, N+$ $2\} \cap \mathcal{N} \neq \emptyset$. In other words, the activation of $N+1$ and $N+2$ is optimized independently from the other $N$ links, regardless whether or not there is a deadline. When deadline is not present, the minimum schedule length equals that for the $N$ links plus one, the latter amounts to the use of group $\{N+1, N+2\}$. Consider introducing deadline for link $N+1$, with $T_{N+1} \in\left[\frac{2}{3}, 1\right)$. With this setting, the deadline constraint is clearly non-redundant, i.e., the optimum schedule length has to increase. Moreover, the problem is feasible, and the new minimum schedule length equals that for the $\mathcal{N}$ links plus a constant that is easily derived from the value of $T_{N+1}$. Therefore, any algorithm solving the instance with the deadline also gives the minimum time schedule for the $N$ links without deadline, and the theorem follows.

\section{REFERENCES}

[1] A. Capone, I. Filippini, S. Gualandi, and D. Yuan, "Resource optimization in multi-radio multi-channel wireless mesh networks," in Mobile Ad Hoc Networking: Cutting Edge Directions, 2nd ed., S. Basagni, M. Conti, S. Giordano, and I. Stojmenovic, Eds. Wiley and IEEE Press, 2013, pp. 241-274.

[2] A. Pantelidou and A. Ephremides, "Scheduling in wireless networks," Found. Trends. Network., vol. 4, no. 4, pp. 421-511, 2009.

[3] L. Georgiadis, M. J. Neely, and L. Tassiulas, "Resource allocation and cross-layer control in wireless networks," Found. Trends. Network., vol. 1 , no. 1, pp. 1-144, 2006

[4] R. Nelson and L. Kleinrock, "Spatial TDMA: a collision-free multihop channel access protocol," IEEE Trans. Commun., vol. 33, no. 9, pp. 934-944, 1985.

[5] E. Arikan, "Some complexity results about packet radio networks," IEEE Trans. Inf. Theory, vol. 30, no. 4, pp. 681-685, 1984.

[6] S. Ramanathan and E. L. Lloyd, "Scheduling algorithms for multihop radio networks," IEEE/ACM Trans. Netw., vol. 1, no. 2, pp. 166-177, 1993.

[7] C. G. Prohazka, "Decoupling link scheduling constraints in multihop packet radio networks," IEEE Trans. Comput., vol. 38, no. 3, pp. 455458, 1989.

[8] P. Gupta and P. R. Kumar, "The capacity of wireless networks," IEEE Trans. Inf. Theory, vol. 46, no. 2, pp. 388-404, 2000.

[9] S. A. Borbash and A. Ephremides, "Wireless link scheduling with power control and SINR constraints," IEEE Trans. Inf. Theory, vol. 52, no. 11, pp. 5106-5111, 2006.

[10] P. Björklund, P. Värbrand, and D. Yuan, "A column generation method for spatial TDMA scheduling in ad hoc networks," Ad Hoc Networks, vol. 2, no. 4, pp. 405-418, 2004

[11] O. Goussevskaia, Y. A. Pswald, and R. Wattenhofer, "Complexity in geometric SINR," in Proc. of ACM MobiHoc, 2007, pp. 100-109.

[12] S. Kompella, J. E. Wieselthier, A. Ephremides, H. D. Sherali, and G. D. Nguyen, "On optimal SINR-based scheduling in multihop wireless networks," IEEE/ACM Trans. Netw., vol. 18, no. 6, pp. 1713-1724, 2010.

[13] V. Angelakis, A. Ephremides, Q. He, and D. Yuan, "Minimum-time link scheduling for emptying backlogged traffic in wireless systems: solution characterization and algorithmic framework," IEEE Trans. Inf. Theory, vol. 60, no. 2, pp. 1083-1100, 2014.

[14] L. Tassiulas and A. Ephremides, "Stability properties of constrained queueing systems and scheduling policies for maximum throughput in multihop radio networks," IEEE Trans. Automat. Contr., vol. 37, no. 12, pp. 1936-1948, 1992.
[15] L. Georgiadis, G. S. Paschos, L. Libman, and L. Tassiulas, "Minimal evacuation times and stability," IEEE/ACM Trans. Netw., vol. 23, no. 3, pp. 931-945, 2015.

[16] Z. Mao, C. E. Koksal, and N. B. Shroff, "Optimal online scheduling with arbitrary hard deadlines in multihop communication networks," IEEE/ACM Trans. Netw., vol. 24, no. 1, pp. 177-189, 2016.

[17] T. Ling and N. B. Shroff, "Scheduling real-time traffic in ATM networks," in Proc. of IEEE INFOCOM, 1996, pp. 198-205.

[18] P. P. Bhattacharya, L. Tassiulas, and A. Ephremides, "Optimal scheduling with deadline constraints in tree networks," IEEE Trans. Autom. Control, vol. 42, no. 12, pp. 1703-1705, 1997.

[19] C. L. Liu and J. W. Layland, "Scheduling algorithms for multiprogramming in a hard-real-time environment," Journal of ACM, vol. 20, no. 1, pp. 46-61, 1973.

[20] Y. Li, H. Zhang, Z. Huang, and M. Albert, "Optimal link scheduling for delay-constrained periodic traffic over unreliable wireless links," in Proc. of IEEE INFOCOM, 2014, pp. 1465-1473.

[21] S. Shakkottai and R. Srikant, "Scheduling real-time traffic with deadlines over a wireless channel," Wireless Netw., vol. 8, no. 1, pp. 13-26, 2002.

[22] G. D. Nguyen, S. Kompella, C. Kam, J. E. Wieselthier, and A. Ephremides, "Minimum-energy link scheduling for emptying wireless networks," in Proc. of IEEE WIOPT, 2015, pp. 207-212.

[23] M. Grötschel, L. Lovász, and A. Schrijver, "The ellipsoid method and its consequences in combinatorial optimization," Combinatorica, vol. 1, no. 2, pp. 169-197, 1981.

Qing He (S'11-M'16) received her BSc and MSc degrees in Electrical Engineering from Nanjing University, China, in 2001 and 2004, respectively, and her PhD degree from the Department of Science and Technology, Linköping University, Sweden, in 2016. She is currently a Post-Doctoral Researcher with the School of Electrical Engineering and Computer Science, KTH Royal Institute of Technology, Sweden. She has a second degree in Finance and had been working as a software designer and system engineer in Lucent Technologies Bell Labs until 2011. Her research interests are in the areas of applied optimization, information theory, and machine learning, with applications to wireless systems.

Di Yuan (M'03-SM'15) received his MSc degree in Computer Science and Engineering, and $\mathrm{PhD}$ degree in Optimization at Linköping Institute of Technology in 1996 and 2001, respectively. He is full professor in telecommunications at the Department of Science and Technology, Linköping University, and head of a research group in mobile telecommunications. His current research mainly addresses network optimization of $4 \mathrm{G}$ and $5 \mathrm{G}$ systems, and capacity optimization of wireless networks. Dr Yuan has been guest professor at the Technical University of Milan (Politecnico di Milano), Italy, in 2008, and senior visiting scientist at Ranplan Wireless Network Design Ltd, United Kingdom, in 2009 and 2012. In 2011 and 2013 he has been part time with Ericsson Research, Sweden. In 2014 and 2015 he has been visiting professor at the University of Maryland, College Park, MD, USA. He is an area editor of the Computer Networks journal. He has been in the management committee of four European Cooperation in field of Scientific and Technical Research (COST) actions, invited lecturer of European Network of Excellence EuroNF, and Principal Investigator of several European FP7 and Horizon 2020 projects. He is a co-recipient of IEEE ICC'12 Best Paper Award, and supervisor of the Best Student Journal Paper Award by the IEEE Sweden Joint VT-COM-IT Chapter in 2014.

Anthony Ephremides (M'71-SM'77-F'84-LF'09) received the Ph.D. degree in electrical engineering from Princeton University, Princeton, NJ, USA, in 1971. He holds the Cynthia Kim Professorship of Information Technology with the Electrical and Computer Engineering Department, University of Maryland, College Park, MD, USA, where he is a Distinguished University Professor and has a joint appointment with the Institute for Systems Research, of which he was among the founding members in 1986. He has been with the University of Maryland since 1971. He is the author of several hundred papers, conference presentations, and patents, and has received numerous awards, including the IEEE Third Millennium Medal, the 2000 Outstanding Systems Engineering Faculty Award from the Institute for Systems Research, the Kirwan Faculty Research and Scholarship Prize from the University of Maryland in 2001, and the 2006 Aaron Wyner Award for Exceptional Service and Leadership to the IEEE Information Theory Society. His research interests lie in the areas of communication systems and networks and all related disciplines, such as information theory, control and optimization, wireless networks, and energy efficient systems. 